\title{
ALTERNATIVE VARIATIONAL FORMULATIONS FOR FIRST ORDER PARTIAL DIFFERENTIAL SYSTEMS*
}

\author{
BY \\ NIMA GEFFEN \\ University of California, Los Angeles
}

\begin{abstract}
Two simple alternative variational principles are derived for a first order differential system with appropriate initial and boundary conditions. The problem is assumed to be well posed, and may be nonlinear, nonhomogeneous and of any type (i.e. elliptic, hyperbolic or mixed). Primitive variables are used, which allows for non-smooth solutions. Redundancy in the system is considered, and applications to fluidynamics and electrodynamics fields given.
\end{abstract}

1. Introduction. Two forms of mathematical modeling of physical continua are possible: (i) a system of first order, partial differential equations for the field components and quantities; (ii) an integral representation of the field via a variational principle, where a functional of the field quantities becomes stationary at the solution of the differential system which constitutes its Euler-Lagrange equations. The two approaches are common in physics (e.g. the Newtonian and Lagrangian representations of mechanics, Hamilton's principle of least action, Fermat's principle in optics) and both have been extremely useful in many and varied areas, both in classical (e.g. mechanics, electrodynamics, relativity e.g. [1-3]) and quantum applications [4].

The common variational formulations (e.g. [1-4]) in theoretical physics are used to derive the Euler differential equations and ignore initial conditions and the question of well posedness. The principle of least action in mechanics is formulated as a boundary value problem, where the initial and final locations are prescribed, while, in fact, it is an initial value problem where locations and velocity are given initially and evolve according to the laws of motion. The same holds for Fermat's principle in optics and is true for the standard variational representation of the electromagnetic field, relativity, and quantum electrodynamics.

For the complete resolution (analytic or numerical) of a particular physical or engineering situation, appropriate initial and boundary conditions have to be considered, the

\footnotetext{
${ }^{*}$ Received July 21, 1982. Work supported, in part, by AF Grant AFOSR-81-0017.

The author's permanent address is Department of Applied Mathematics, Tel-Aviv University, Remant-Aviv, 69978, Israel. 
problem has to be well posed, i.e. to be solvable uniquely and stably, and the model has to encompass relevant geometries and non-smoothness of the solution (e.g. shocks).

Initial and boundary conditions are incorporated in the variational formulations below as well as given sources and vorticity distributions for the field. Some of the formulations, we believe, are new.

The initial motivation for this work came from finite-element [5] solution of problems of mixed type related to transonic aerodynamics. It shares common points with a recent analysis of similar (albeit linear) field equations [6] and is related, we believe, to other recent approaches (e.g. [7]) and to finite-element formulations and analysis of analogous continuum systems of engineering importance [10-12].

2. General equations. Continuum mechanics, electrodynamics and other physical systems can be modelled by various specializations of the following set of first order, partial differential equations, specifying the source distribution of a vector field $\mathbf{A}$ :

$$
\nabla \cdot \mathbf{A}=G
$$

and the curl of the vector field $\mathbf{u}$ :

$$
\nabla \times \mathbf{u}=\mathbf{W}
$$

for $n+1$ independent variables:

(i) $\mathbf{x}=\left(x_{0}, x_{1}, \ldots, x_{n}\right), n \leqslant 3$, where $x_{0}$ may designate time (or a timelike coordinate). $m$ dependent variables $(m \leqslant 3)$ :

(ii) $\mathbf{u}=\mathbf{u}(\mathbf{x})=\left(u_{1}, \ldots, u_{m}\right)\left(x_{0}, x_{1}, \ldots, x_{n}\right)$ and:

(iii) $\mathbf{A}=\mathbf{A}(\mathbf{x}, \mathbf{u})=\left(A_{0}, \ldots, A_{n}\right)$.

$G=G(\mathbf{x}, \mathbf{u})$ is the source distribution for the vector field $\mathbf{A}$. $\mathbf{W}=\mathbf{W}(\mathbf{x}, \mathbf{u})=W$ is the vorticity distribution for the 'solution' field $\mathbf{u}$. If $\mathbf{u}$ appears linearly in (iii), i.e.:

$$
\frac{\partial \mathbf{A}}{\partial \mathbf{u}}=\frac{\partial A_{i}}{\partial u_{j}}=c_{i j}(x)
$$

where the $c_{i j}(\mathbf{x})$ do not depend on $\mathbf{u}$, which in addition, does not appear in (iv) i.e.: $G=G(\mathbf{x})$ the system (1), (2) is linear. If $G=G(\mathbf{x}, \mathbf{u})$ the system is semi-linear. If $\mathbf{u}$ appears in A nonlinearly, i.e.:

$$
\frac{\partial \mathbf{A}}{\partial \mathbf{u}}=\frac{\partial A_{i}}{\partial u_{j}}=f_{i j}(\mathbf{u}, \mathbf{x})
$$

the system is quasilinear.

The vorticity $\mathbf{W}$ has to satisfy a compatibility condition,

$$
\nabla \cdot \mathbf{W}=0
$$

and thus not all vorticity distributions are admissible.

The system (1), (2) can be extended to coupled fields $\mathbf{u}(x), \mathbf{v}(x)$ :

$$
\begin{aligned}
\nabla \cdot \mathbf{A}_{l}=G_{l} \quad l=1,2, \ldots, \\
\nabla \times \mathbf{u}=\mathbf{W}, \\
\nabla \times \mathbf{v}=\mathbf{\Omega},
\end{aligned}
$$




$$
\begin{aligned}
& \nabla \cdot \mathbf{W}=0, \\
& \nabla \cdot \mathbf{\Omega}=0,
\end{aligned}
$$

where $\mathbf{A}_{l}=\mathbf{A}_{l}(\mathbf{x}, \mathbf{u}, \mathbf{v})$, e.g. the electromagnetic field equations.

The system of Eqs. (1), (2) is quite general, it can be linear and nonlinear, elliptic, hyperbolic or mixed, with smooth or nonsmooth solutions. The independent variables $x_{i}$ may designate space and time coordinates, and different kinds of initial and/or boundary conditions may be appropriate for different problems. Higher order equations may be put into this form, and applications include problems from electro-magnetic field theory, fluidynamics, and plasma-dynamics, including flows with shocks.

A unique solution to a field governed by equations (1), (2) is depicted for appropriate initial/boundary conditions, where a number (possibly all) of the field components $u_{i}$ are given on parts (possibly all) of the boundary $\partial \Omega_{l}=\Sigma_{j=1}^{N} \partial \Omega_{j}$ of the region $\Omega$ occupied by the field; i.e.,

$$
\tilde{\mathbf{u}}_{i}(x)=\mathbf{f}_{i}(x) \quad \text { on } \partial \Omega_{l}, \quad 1 \leqslant l \leqslant N .
$$

The specific form of (3) depends on the problem and on the type of the partial differential system (1), (2) (e.g. pure boundary values for time-independent elliptic problems, initial values for the Cauchy hyperbolic case, appropriate 'mixed' conditions for equations of mixed type).

3. Variational formulations. A variational formulation of the field $\mathbf{u}(x)$ satisfying (1), (2), (3) is a functional $J(v)$ defined on a domain $\Omega$ whose stationary value is obtained for $\mathbf{v}=\mathbf{u}$ :

$$
\delta J(\mathbf{v})=0 \Leftarrow \mathbf{v}=\mathbf{u} .
$$

For well-posed problems, for which $\mathbf{u}(\mathbf{x})$ is unique, we have:

$$
\delta J(\mathbf{v})=0 \Leftrightarrow \mathbf{v}=\mathbf{u} .
$$

Variational formulations are scalar, short, additive (the functionals for complex systems are direct sums of their simpler parts), invariant under appropriate classes of transformations and are often convenient for theoretical analysis and for numerical simulations, e.g. by finite differences and finite elements; the integrals can be discretized and approximated, and smoothness requirements on the functions are less stringent than for the corresponding differential system. This last point is most important from the numerical view point and for the treatment of shocks.

The case $G=0$ is treated in [8]. The functional $J(\mathbf{v}, \mathbf{x})$ :

$$
J=\int_{\Omega}[L(\mathbf{v}, \mathbf{x})+\lambda,(\nabla x \mathbf{v}-\mathbf{W})] d \mathbf{s}+\int_{\partial \Omega_{m}} \lambda x \mathbf{v} \cdot \mathbf{d} \boldsymbol{\sigma}
$$

is stationary at $\mathrm{v}=u$ :

$$
\delta J(\mathbf{v})=0, \mathbf{v}=\mathbf{u}
$$


provided that

$$
\begin{aligned}
\nabla_{\mathbf{u}} x \mathbf{A} & =0, \\
\mathbf{A} & =\nabla_{\mathbf{u}} L, \\
\mathbf{A} & =-\nabla x \boldsymbol{\lambda} .
\end{aligned}
$$

The variation is done on all $\mathbf{v}$ for which $J$ is defined, and boundary conditions are satisfied on the boundary $\partial \Omega_{i}=\partial \Omega-\partial \Omega_{l}$ the conditions on the part $\partial \Omega_{l}$ of the boundary is "free".

For the nonsourceless (or sourceful) case, the following variational statements hold:

THEOREM 1. The functional

$$
J(\mathbf{v}, \boldsymbol{\lambda})=\int_{\Omega}[L-\mathbf{g} \cdot \mathbf{v}+\boldsymbol{\lambda} \cdot(\nabla x \mathbf{v}-W)] \cdot d \mathbf{x}+\int_{\partial \Omega_{m}} \lambda x \mathbf{v} \cdot d \boldsymbol{\sigma}
$$

is stationary for $\mathbf{v}=\mathbf{u}$ satisfying (1), (2), (3) provided that

$$
\nabla_{\mathbf{u}} x \mathbf{A}=0
$$

where

$$
\begin{aligned}
\nabla \cdot \mathbf{g} & =G \text { or } \mathbf{g}=\nabla^{-1} G, \\
\mathbf{A} & =\nabla_{\mathbf{u}} L, \\
\mathbf{A} & =-\nabla x \boldsymbol{\lambda}+\mathbf{g}
\end{aligned}
$$

$\mathbf{v}$ is allowed to vary over all functions for which (9) is defined and finite, and which satisfy the coercive boundary conditions (3). In this formulation $\mathbf{v}$ is required to be differentiable while $\boldsymbol{\lambda}$ can be just integrable.

Proof. An arbitrary variation of $\mathbf{v}$ and $\boldsymbol{\lambda}$ in $J(\mathbf{v}, \boldsymbol{\lambda})(25)$ leads to

$$
\begin{aligned}
\delta J= & \int_{\Omega}[\delta \mathbf{L}-\mathbf{g} \cdot \delta \mathbf{v}+\delta \boldsymbol{\lambda} \cdot(\nabla x \mathbf{v}-\mathbf{W})+\boldsymbol{\lambda} \cdot \nabla x \delta \mathbf{v}] d x \\
& +\int_{\partial \Omega} \delta \boldsymbol{\lambda} x \mathbf{v} \cdot d \sigma+\int_{\partial \Omega} \lambda x \delta \mathbf{v} \cdot \mathbf{d} \boldsymbol{\sigma} .
\end{aligned}
$$

Substituting

$$
\begin{aligned}
\delta L & =\nabla_{\mathbf{v}} L \cdot \delta \mathbf{v}, \\
\lambda \cdot \nabla x \delta \mathbf{v} & =\delta \mathbf{v} \cdot \nabla x \lambda-\nabla \cdot(\lambda x \delta \mathbf{v})
\end{aligned}
$$

we get

$$
\begin{aligned}
\delta J= & \int_{\Omega}\left[\left(\nabla_{\mathbf{v}} L-\mathbf{g}+\nabla x \boldsymbol{\lambda}\right) \cdot \delta \mathbf{v}+(\nabla x \mathbf{v}-\mathbf{W}) \cdot \delta \boldsymbol{\lambda}\right] d \mathbf{x} \\
& -\int_{\partial \Omega} \lambda x \delta \mathbf{v} \cdot d \boldsymbol{\sigma}+\int_{\partial \Omega} \delta \boldsymbol{\lambda} x \mathbf{v} \cdot \mathbf{d} \boldsymbol{\sigma}+\int_{\partial \Omega} \lambda x \delta \mathbf{v} \cdot d \boldsymbol{\sigma}
\end{aligned}
$$

Necessary conditions for stationarity of $J(\mathbf{v}, \lambda)$

$$
\delta J=0 \quad \text { at } \mathbf{v}=\mathbf{u}
$$


are

$$
\nabla_{\mathbf{u}} L-\mathbf{g}=-\nabla x \boldsymbol{\lambda}
$$

i.e.,

$$
\begin{aligned}
& \mathbf{A}=\nabla_{\mathbf{u}} L, \quad \nabla \circ \mathbf{A}=\nabla \circ \mathbf{g}, \\
& \mathbf{A}=-\nabla x \boldsymbol{\lambda}+\mathbf{g},
\end{aligned}
$$

and in addition:

$$
\int \delta \lambda x \mathbf{v} \circ d \boldsymbol{\sigma}=0
$$

on the free boundary, as stated.

Remark. Natural boundary conditions for the functional (9) without the surface term are: $\boldsymbol{\lambda}=0$ or $\boldsymbol{\lambda} \| \mathbf{d} \boldsymbol{\sigma}$ on the 'free' boundary (where $\mathbf{v}$ is not prescribed).

An alternative formulation is obtained by integrating the second term by parts, in the RHS of e.g. (9) using the vector identity:

$$
\nabla \cdot(\boldsymbol{\lambda} x \mathbf{v})=\mathbf{v} \cdot \nabla x \boldsymbol{\lambda}-\boldsymbol{\lambda} \cdot \nabla x \mathbf{v}
$$

substituting in (9) for $\lambda \cdot \nabla x \mathbf{v}$, we obtain

THEOREM 2. The functional

$$
J(\mathbf{v}, \boldsymbol{\lambda})=\int(L-\mathbf{g} \cdot \mathbf{v}+\mathbf{v} \cdot \nabla x \boldsymbol{\lambda}-\boldsymbol{\lambda} \cdot \mathbf{W}) \mathbf{d x}
$$

is stationary for $\mathbf{v}=\mathbf{u}$ satisfying (1), (2).

In the variational formulation (14), the Lagrange multiplier $\boldsymbol{\lambda}$ is required to have integrable first derivatives, which appear explicitly in $J$, while $\mathbf{v}$ can just be integrable.

The surface term drops out, and the solution $\mathbf{v}=\mathbf{u}$ satisfies the natural boundary conditions:

$$
\boldsymbol{\lambda} x \mathbf{u} \cdot \mathbf{d} \boldsymbol{\sigma}=0 \text { on } \partial \Omega
$$

i.e., either

$$
\boldsymbol{\lambda} \| \mathbf{u} \text { or } \mathbf{u} \| \mathbf{d} \boldsymbol{\sigma}
$$

i.e., $u \| \hat{n}$ on $\partial \Omega$.

\section{Examples.}

(a) Steady electromagnetic field. Maxwell's equations for a steady state can be written as [3]:

(i) $\nabla \cdot \mathbf{E}=\rho$,

(ii) $\nabla x \mathbf{E}=0$,

(iii) $\nabla \cdot \mathbf{B}=0$,

(iv) $\nabla x \mathbf{B}=\mathbf{j}$.

A variational statement (9) for (i), (ii) is

$$
J\left(\mathbf{E}, \lambda^{E}\right)=\int_{\Omega}\left[\left(E^{2} / 2-\mathbf{g} \cdot \mathbf{E}\right)+\lambda^{(E)}(\nabla x \mathbf{E})\right] \mathbf{d x}+\int_{\partial \Omega_{k}} \lambda^{(E)} x \mathbf{E} \cdot \mathbf{d} \boldsymbol{\sigma}
$$


for $\mathbf{E}$ arbitrary, or

$$
J(\mathbf{E})=\int_{\Omega}\left(E^{2} / 2-\mathbf{g} \cdot \mathbf{E}\right) d x
$$

for $\mathbf{E}$ irrotational where $\mathbf{g}$ is any solution to

$$
\nabla \cdot \mathbf{g}=\boldsymbol{\rho} \text { or } \mathbf{g}=\nabla^{-1} \rho .
$$

The variational statement (14) for (i), (ii) becomes:

$$
J\left(\mathbf{E}, \lambda^{E}\right)=\int_{\Omega}\left[\left(E^{2} / 2-\mathbf{g} \cdot \mathbf{E}\right)-\mathbf{E} \cdot\left(\nabla x \lambda^{(E)}\right)\right] .
$$

The corresponding variational formulations for the magnetic equations (iii), (iv) are

$$
J\left(\mathbf{B}, \lambda^{B}\right)=\int\left[B^{2} / 2+\lambda^{(B)}(\nabla x \mathbf{B}-\mathbf{j})\right] \mathbf{d x}+\int_{\partial \Omega_{m}} \lambda^{(B)} x \mathbf{B} \cdot \mathbf{d} \boldsymbol{\sigma}
$$

and

$$
J(\mathbf{B})=\int_{\Omega}\left[B^{2} / 2-\mathbf{B} \cdot\left(\nabla x \lambda^{(B)}\right)\right] .
$$

The functionals for the combined field are obtained by simply adding the ones for the 'separated' system; $\left(9^{\prime}\right)$ and $\left(9^{\prime \prime}\right)$ give

$$
\begin{aligned}
J\left(\mathbf{E}, \mathbf{B} ; \lambda^{E}, \lambda^{B}\right)= & \int_{\Omega} \frac{E^{2}-B^{2}}{2}-\mathbf{g} \cdot \mathbf{E}+\lambda^{(B)} \cdot \mathbf{j}+\lambda^{(E)} \cdot(\nabla x \mathbf{E})-\lambda^{(B)} \cdot(\nabla x \mathbf{B}) \mathbf{d x} \\
& +\int_{\partial \Omega_{k}^{E}} \lambda^{E} x \mathbf{E} \cdot \mathbf{d} \boldsymbol{\sigma}-\int_{\partial \Omega_{i}^{B}} \lambda^{B} x \mathbf{B} \cdot \mathbf{d} \boldsymbol{\sigma} .
\end{aligned}
$$

While (14') and (14) combine to

$$
J\left(\mathbf{E}, \mathbf{B} ; \lambda^{E}, \lambda^{B}\right)=\int \frac{E^{2}-B^{2}}{2}-g \cdot \mathbf{E}-\mathbf{E} \cdot \nabla x \lambda^{E}+\mathbf{B} \cdot \nabla x \lambda^{B} d x .
$$

(b) Steady fluidynamics. The differential equations are

$\nabla \cdot(\rho \mathbf{q})=0$,

$\rho=\rho\left(q^{2}\right)$,

$\nabla \times \mathbf{q}=\mathbf{W}$

and the corresponding Lagrangian $L$ and functionals are:

$$
\begin{gathered}
\rho \mathbf{u}=\frac{\partial L}{\partial \mathbf{u}} \quad \text { or } \quad \rho u_{i}=\frac{\partial L}{\partial u_{i}}, \\
J(\mathbf{u}, \boldsymbol{\lambda})=\int_{\Omega}[L+\lambda \cdot(\nabla x \mathbf{u}-\mathbf{W})] \mathbf{d x}+\int_{\partial \Omega} \lambda x \mathbf{u} \cdot \mathbf{d} \boldsymbol{\sigma}
\end{gathered}
$$

and

$$
J(\mathbf{u}, \lambda)=\int_{\Omega} L-\lambda \cdot W+\mathbf{u} \cdot \nabla x \boldsymbol{\lambda}
$$

for the appropriate function spaces, with the required smoothness properties and constraints, in the region and on the boundary. 
The results (16) have been described and analyzed in $[8,9]$ where applications to specific flows are given. While (16) requires that $\mathbf{u}$ be smoother than $\lambda$, the opposite holds for (17), which is more in accord with the physics of the problem, where the field variables $\mathbf{u}$ are related to the derivatives of the 'vector potential' $\boldsymbol{\lambda}$.

5. Redundancy in differential and variational formulations. Variational formulation have been widely used for approximating field solutions, notably by the Ritz and finite elements methods. The tacit assumption is that the variational problem is well posed, for which a necessary condition is that so is its system of Euler equations and initial boundary conditions; a necessary condition in turn, for stable solvability is that it be non-redundant. The differential system, however, is redundant. The system (1), (2), includes $(n+1)$ first order partial differential equations for the $n$ unknown components of the dependent vector $\mathbf{u}$. The $n$ equations (2), however, are not linearly independent. In order to solve a problem, the system has to be reduced to a 'posed' system, for which number of equations has to be equal to the number of unknowns.

The variational formulations Eqs. (9), (14) are redundant in the sense that their Euler Eqs. (1), (2) are redundant. Since one of the rotationality conditions (2) is implied by the rest, its corresponding $\boldsymbol{\lambda}$ component is redundant. This can also be inferred from Eq. (13) which is analogous to (2) (with $\mathbf{u}$ replaced by $\boldsymbol{\lambda}$ and $\mathbf{W}$ by $(\mathbf{A}-\mathbf{g})$ ), where the system (13) is not independent, and one of the equations results from the others.

his redundancy in the variational formulations is less apparent than in the differential system (where a simple count of equations and unknowns signals a red light-to stop and look). In the usual approximations (e.g., by the Ritz or finite element method) the number of equations is made to equal the number of unknowns, obscuring the fact that the system may have no solution (for inadmissible W not satisfying Eq. (2a)). Simple round-off inaccuracies may turn various procedures unstable. This, we believe, may be one of the causes for instability experienced in various mixed and hybrid finite elements procedures (e.g. [10]-[12]).

Two remedies are suggested:

(i) to remove one rotationality condition, the one in the time-like direction (or mostly so for nonlinear problems);

(ii) to add another term to the functional:

$$
\delta \nabla \circ \mathbf{w}
$$

to enforce the compatibility of the $w$ components and correct any deviation thereof. While (i) removes one of the unknowns (but demands attention in choosing the correct one to eliminate), (ii) adds an unknown-another Lagrange multiplier-which is a drawback. This, however, may be used as a check for simple bench-mark problems.

\section{REFERENCES}

[1] L. D. Landau and E. M. Lifschitz, Mechanics and electrodynamics, a shorter of theoretical physics, Vol. 1, Pergamon Press, 1st ed., 1972

[2] H. Goldstein, Classical mechanics, Addison-Wesley, Second Edition, 1980 
[3] R. P. Feymann, Lectures on physics, California Institute of Technology Notes, 1965, The principle of least action, pp. 19-1-19-14

[4] R. P. Feynman and A. R. Hibbs, Quantum mechanics and path integrals, McGraw-Hill, New York, 1965

[5] N. Geffen, Finite elements for fluidynamics, Final Scientific Report, Grant No. AFOSR-77-3345, 15-18, July 1979

[6] Milton E. Rose, A numerical scheme to solve div $\mathbf{u}=0$, curl $\mathbf{u}=\zeta$, ICASE Report No. 82-8, April 7, 1982

[7] O. Buneman, Ideal gas dynamics in Hamiltonian form with benefit for numerical schemes, Phys. Fluids, to be published; also a presentation at the 7th International Meeting on Numerical Methods in Fluid Dynamics, Stanford University, June 1980

[8] Nima Geffen, $A$ variational formulation for constrained quasilinear vector systems, Quart. Appl. Math. 375-381, October 1977

[9] __ Variational formulations for nonlinear wave propagation and unsteady transonic flow, ZAMP, 28, 1038-1043, 1977

[10] H. A. Meng and R. H. Gallagher, A critical assessment of the simplified hybrid displacement method, Internat. J. Numerical Methods in Engineering, 11, 145-167 (1977), Further comments on the simplified hybrid displacement method, a discussion on the paper above, Ibid., 12, 1457-1484 (1978)

[11] E. Haugeneder and H. A. Meng, Admissible and inadmissible simplifications of variational methods in finite element analysis, presented at the IUTAM Symposium on Variational Methods in Mechanics of Solids, Northwestern Univ., Evanston, Ill., Sept. 11-13, 1978

[12] On an improper modification of a variational principle for finite element plate analysis, ZAMM 59, $637-540(1979)$ 